\title{
Vascular Aging and Cognitive Dysfunction: Silent Midlife Crisis in the Brain?
}

\author{
Chen-Hua Lin ${ }^{a}$ Hao-Min Cheng ${ }^{a-e}$ Shao-Yuan Chuang ${ }^{f}$ \\ Chen-Huan Chen ${ }^{a}, b, d, e$ \\ anstitute of Public Health and ${ }^{b}$ Faculty of Medicine, National Yang-Ming University, \\ ${ }^{c}$ Center for Evidence-Based Medicine, and Departments of ${ }^{\mathrm{d}}$ Medical Education and \\ e Internal Medicine, Taipei Veterans General Hospital, Taipei, and ${ }^{\mathrm{f}}$ National Health \\ Research Institutes, Miaoli, Taiwan, ROC
}

\section{Keywords}

Blood pressure $\cdot$ Brain $\cdot$ Cognition $\cdot$ Middle age $\cdot$ Vascular aging

\begin{abstract}
Background: Vascular aging may cause cerebral microvascular damage and cognitive dysfunction. There is incremental evidence that consistently implicates arterial stiffness being involved in the manifestation of cognitive impairment in the elderly. However, few investigations have examined the relationship between arterial stiffness and cognitive impairment in midlife. Summary: Past studies inconsistently showed improved cognitive outcomes after antihypertensive therapy in elderly populations. Nevertheless, recent findings revealed that blood-pressurelowering treatment in young adults might eliminate or halt the progression of the detrimental effects related to arterial stiffness, indicating that younger adults may have more favorable outcomes in cognition than their older counterparts if early intervention is conducted the subclinical stage. Stiffening of the aorta may lead to an excessive flow pulsatility in the brain that may cause microvascular structural brain damage and worse cognitive performance. Recent investigations have suggested that arterial stiffness is likely to trigger initial silent brain damage, possibly preceding midlife, while the manifestation of cognitive decline and deterioration can be foreseen in the subsequent life span. Key Message: Despite the recent novel findings, definite conclusions on causality between vascular aging and cognitive dysfunction cannot be drawn at present. Further well-powered longitudinal studies with superior neuroimaging indicator, vascular mechanical biomarkers, and sensitive cognitive assessment tools that examine a broad range of age populations may help extend our understanding of the association between vascular aging and cognitive dysfunction throughout the life span.
\end{abstract}




\section{Introduction}

Vascular aging, coupled with enhanced pulsatile hemodynamics, has been shown to be a major independent determinant of cerebral microvascular damage and cognitive function impairment [1,2]. Over a decade, many investigations have suggested that marked arterial stiffness is associated with impaired cognitive function in elderly populations [1, 3-6]. Recently, for middle-aged individuals, a reciprocal interaction between high blood pressure (BP) and poor cognitive performance was suggested [7-10]. Vascular aging is acknowledged as the major pathophysiology for age-related hypertension [11]; however, the mechanisms by which vascular aging leads to cognitive dysfunction in midlife remain poorly understood. Full elucidation of the mechanisms by which the pulsatile hemodynamics may affect the development of cognitive dysfunction starting at a young age is crucial to safeguard vascular health and brain health in midlife [12].

\section{Hypertension and Cognitive Dysfunction in Midlife}

Elevated BP level is associated with cognitive decline and increased risk of dementia [9, $10,12-14]$. It is clear that hypertension may disrupt the cerebral microvascular structure and function [12] and may also be associated with increased risk of ischemic damage of white matter regions critical for cognitive function $[9,15]$. Treatment of hypertension may help prevent or slow down the development of vascular brain lesions and improve cognitive performance in hypertensives $[16,17]$. However, results from clinical trials and randomized trials on the relationship between antihypertensive therapy and development of dementia were inconsistent, and the evidence that antihypertensive treatment improves cognition is not conclusive $[9,12,18]$.

The 17-year follow-up data from 668 community-dwelling Japanese individuals (the Hisayama study) demonstrated that midlife hypertension increased the risk of dementia in late life, regardless of BP level in late life [19]. Consistent with Japanese studies, a western study found that midlife hypertension, whether treated or not, was associated with worse cognitive performance after 20 years of follow-up [20]. These results may imply that the BP-lowering therapy has little reversing effect on the hypertension-related brain damage and does not provide a cognitive protection effect [9].

However, it is speculated that pulsatile hemodynamics, by increasing pressure and/or flow pulsatility, may impose a large pulsatile stress in the brain early in the life span before the incidence of hypertension [21,22]. For example, aortic stiffness, measured by carotidfemoral pulse wave velocity (cfPWV) was found to be strongly associated with incident hypertension in the Framingham cohort [11]. In a recent study, it was found that lowering of the BP level is associated with more normal cfPWV values in young adults, indicating that the elastic and flexible aorta with higher arterial compliance may be more likely to revert to normal and possibly be able to sustain the harmful effects on cognition at a younger age [22]. This may partly explain why medical therapy targeting hypertension alone may not be sufficient to eliminate the detrimental effects on the cerebral microcirculation resulting from vascular aging in the elderly. Since pulsatile hemodynamics is related to BP progression and precedes the development of hypertension [11], arterial stiffness, measured by cfPWV, is a superior predictor of cognitive decline compared to hypertension [23]. This may suggest that attention should be focused on vascular markers, such as cfPWV, to identify individuals at greatest risk of hypertension progression and cognitive dysfunction.

Early intervention or treatment for hypertension in middle age, even in the subclinical state, may be substantially beneficial, given the strong evidence of a deleterious influence of 
midlife hypertension on late-life cognitive function [14, 24]. In fact, even in prehypertensive (systolic BP $>120$ and $<140 \mathrm{~mm} \mathrm{Hg}$ or diastolic BP $>80$ and $<90 \mathrm{~mm} \mathrm{Hg}$ ) middle-aged adults, an elevated systolic BP level was shown to be negatively associated with cognitive performance, including memory and executive function [8]. In a cohort study, prehypertension in midlife was also shown to be associated with increased risk of developing dementia [25]. Another study found significantly worse performance on verbal episodic memory for untreated hypertensive women; even at the prehypertensive level, the predictive performance on memory domain is less satisfactory later in life [7]. All these findings taken together indicate that reducing midlife BP, even at the prehypertensive level, may be beneficial for protecting against subsequent cognitive dysfunction and the development of dementia in later life [7, 8]. Further well-founded longitudinal studies are warranted to clarify the causal relationships between arterial stiffness, BP level, and cognitive impairment before midlife by extending the study population from the elderly to the middle-aged or even young adult population.

\section{Vascular Aging and the Link between the Heart and the Brain}

The brain receives abundant blood supply, by as much as $20 \%$ of cardiac output [26]. With lower impedance in the cerebral microcirculation, the brain is sensitive to the harmful effects of excessive pressure and flow pulsatility resulting from vascular aging [1]. Vascular aging, characterized by increased arterial stiffness and arterial wave reflections, has a significant adverse impact on the cerebral vascular system, leading to cerebral microvascular ischemia and structural changes and subsequent premature cognitive dysfunction later in life [27-29].

Previously, for the older population, the community-based Age, Gene/Environment Susceptibility - Reykjavik Study found that cfPWV was associated with higher white matter hyperintensity volume, a common imaging evidence of cerebral injury [1]. Moreover, carotid pulsatility index, pulse pressure, and cfPWV were each associated with increased risk of silent subcortical infarcts [1]. Altogether, the results strongly support that stiffening of the aorta is associated with transmission of excessive flow pulsatility into the brain, leading to progressive microvascular structural brain damage in the elderly [27].

In addition, the carotid pulsatility index was associated with lower whole-brain gray and white matter volumes, indicative of reduced mean flow concomitant with increased flow pulsatility in the cerebral circulation [1]. Consequently, increased cerebrovascular resistance and flow pulsatility may restrict the blood flow in the brain, leading to overall brain atrophy and consequential adverse impacts on multiple cognitive performances. This may explain why the carotid pulsatility index was found to be associated with a broad range of cognitive domain declines, including lower memory scores, slower processing speed, and worse performance on tests assessing executive function, whereas cfPWV and carotid pulse pressure were each associated with lower memory scores only [1].

The underlying mechanism the vascular aging-associated cardio-cerebral interaction has not been clearly delineated. Compared to the carotid arteries, the aorta is highly compliant in young and healthy subjects. Such an impedance "mismatch" constitutes barriers of wave reflections when a traveling pressure wave from the ascending aorta encounters the firstgeneration arteries, meaning that the pulsatile energy will not be fully transmitted into the distal vasculature [1]. After midlife, progressive impedance "matching" due to a disproportionate increase in aortic impedance may dissipate the aorta-carotid interface, increase the excessive pressure and flow pulsatility into the cerebral circulation, and subsequently result in microvascular damage and remodeling as well as poor cognitive function [1]. Therefore, increased aortic stiffness, being associated with cerebral ischemia, tissue damage, and cognitive decline, tightens the link between cardiac and cerebral structure and function. 
Recently, attention has been paid to the younger population because it is likely that the negative effect of greater aortic stiffness and pulsatility can have a cumulative effect, initiating a silent impact on the brain earlier in the life span $[21,30]$. The Framingham Third Generation Cohort Study found that in younger adults aged between 30 and 45 years, only a single marker of cerebral atrophy was associated with cfPWV, whereas in midlife individuals (age 45-65 years), cfPWV was associated with more advanced brain injury, evident by increased white matter hyperintensities in brain magnetic resonance imaging and worse cognitive performances in processing speed and executive function [30]. The findings highlighted the possibility that arterial stiffness, causing initial brain damage to the cerebral structure, may begin in young adulthood, before the manifestation of cognitive decline and progressive deterioration in later life [30]. Moreover, cfPWV was consistently shown to be associated with pioneering white matter injury (measured by fractional anisotropy) and regional earlier brain atrophy (measured by gray matter integrity), whereas other hemodynamic parameters, including augmentation index and pulse pressure, showed no significant link to change of brain structure [22]. In the younger population, evidence of earlier microstructural injury prior to white matter hyperintensities was found, further supporting the hypothesis that initial and continuous brain damage related to arterial stiffness may start in or even precede midlife [22]. However, current evidence cannot draw definite conclusions due to the cross-sectional study design of most studies. Future longitudinal analyses are warranted to identify the causation between vascular aging and brain structural and functional changes throughout the life span.

\section{Knowledge Gaps and Recommendations for Future Studies}

Currently, studies have been done on diverse study populations, evaluating different measures of cerebral microvascular change, cognitive function, and arterial stiffness [2, 31]. However, one systemic review and meta-analysis showed that arterial stiffness, measured by cfPWV or brachial-ankle pulse wave velocity (baPWV), was significantly associated with microvascular damage in the brain, whereas the association between arterial stiffness and cognitive impairment was inconclusive due to a relatively limited number of valid studies, as well as inconsistent results [2]. In the midlife population, in order to identify the earliest changes, whether in brain structure or cognition, valid and sensitive mechanical biomarkers and assessment tools play crucial roles. A few potentially valuable markers are recommended for inclusion in future investigations.

Firstly, regarding the neuroimaging markers in identifying brain structural changes, a valuable biomarker derived from magnetic resonance imaging scans, free water (FW), was recently proposed in identifying white matter injury [32]. Previously, FW content was commonly used in detecting cerebral injury in neurologic conditions and disorders, including Parkinson disease and schizophrenia $[33,34]$. In the recent study, a strong association between cfPWV and FW content across a broad range of ages was found [32], indicating that FW may be a more sensitive marker to identify earlier white matter injury related to arterial stiffness in young populations. Compared to fractional anisotropy (an indicator of white matter degeneration) and white matter hyperintensities, excess of white matter FW might be temporary and possibly reversible if early interventions are given, whereas white matter hyperintensities, indicating permanent damage, may have an irreversible impact on cerebral function [32]. Therefore, using FW to detect the changes in the brain early, before symptoms or substantial structural and functional damage manifest, may be beneficial.

Secondly, in addition to commonly used vascular markers (cfPWV, baPWV, augmentation index, and central pulse pressure), arterial reservoir function parameters may have potential value in predicting cognitive change. Vascular aging is characterized not only by accelerated 
wave transmission and more pronounced wave reflections, but also by a reduced reservoir function in the arterial system [35]. Excess pressure integral derived from the reservoir-wave analysis was shown in a cross-sectional study to have a significant inverse association with gray matter volume [36]. However, the relationship between reservoir function parameters and cognitive function has never been comprehensively investigated. Whether these mechanical biomarker should be used as estimates of vascular aging in relation to cognition deserves more investigations.

Finally, in order to identify the earliest change in midlife, valid and sensitive cognitive function assessment tools are needed. Most studies have shown overreliance on the MiniMental State Examination (MMSE) to measure cognitive function [31], which may limit the possibility of detecting minor cognitive change in various domains. The MMSE has a poor sensitivity for distinguishing mild cognitive impairment due to the insufficient complexity and executive function items $[31,37,38]$. Compared to the MMSE, the Montreal Cognitive Assessment (MoCA) instrument is a more challenging test that includes higher-level language as well as more complex executive function and visual-spatial processing to enable detection of mild impairment [39-42]. Therefore, future large-scale longitudinal studies using MoCA are recommended to maximize the chance of identifying earlier change in different cognitive domains and global cognition.

\section{Conclusions}

Vascular aging, characterized by increased arterial stiffness and wave reflection, has been recognized as one of the fundamental mechanisms that contribute to cerebrovascular dysfunction and cognitive decline. Whether the cerebral/cognitive impairment resulting from increased pressure and flow pulsatility starts earlier in the life span (along with the progression of vascular aging and development of hypertension) remains inconclusive. Therefore, future studies extending the study population from elderly life to midlife, and even to younger adults, are warranted to explore the associations between vascular aging and cognition across the life span, and to provide opportunities and targets for effective intervention.

\section{Disclosure Statement}

The authors declare no conflict of interest.

\section{References}

1 Mitchell GF, et al: Arterial stiffness, pressure and flow pulsatility and brain structure and function: the Age, Gene/ Environment Susceptibility - Reykjavik Study. Brain 2011;134(Pt 11):3398-3407.

$\checkmark 2$ vanSloten TT, etal: Association between arterial stiffness, cerebral small vessel disease and cognitive impairment: a systematic review and meta-analysis. Neurosci Biobehav Rev 2015;53:121-130.

-3 Pase MP, et al: Arterial stiffness as a cause of cognitive decline and dementia: a systematic review and metaanalysis. Intern Med J 2012;42:808-815.

4 Poels MM, et al: Arterial stiffness, cognitive decline, and risk of dementia: the Rotterdam Study. Stroke 2007;38: 888-892.

5 Singer J, et al: The association between pulse wave velocity and cognitive function: the Sydney Memory and Ageing Study. PLoS One 2013;8:e61855.

-6 Tsao CW, et al: Relations of arterial stiffness and endothelial function to brain aging in the community. Neurology 2013;81:984-991.

7 Chen KH, et al: Prehypertension in midlife is associated with worse cognition a decade later in middle-aged and older women. Age Ageing 2015;44:439-445.

-8 Jennings JR, et al: Prehypertensive blood pressures and regional cerebral blood flow independently relate to cognitive performance in midlife. J Am Heart Assoc 2017;6:e004856. 
$\$ 9$ Hughes TM, Sink KM: Hypertension and its role in cognitive function: current evidence and challenges for the future. Am J Hypertens 2016;29:149-157.

10 Power MC, et al: The association between blood pressure and incident Alzheimer disease: a systematic review and meta-analysis. Epidemiology 2011;22:646-659.

11 Kaess BM, et al: Aortic stiffness, blood pressure progression, and incident hypertension. JAMA 2012;308:875881.

12 Iadecola C, et al: Impact of hypertension on cognitive function: a scientific statement from the American Heart Association. Hypertension 2016;68:e67-e94.

13 Euser SM, et al: The effect of age on the association between blood pressure and cognitive function later in life. J Am Geriatr Soc 2009;57:1232-1237.

14 Walker KA, Power MC, Gottesman RF: Defining the relationship between hypertension, cognitive decline, and dementia: a review. Curr Hypertens Rep 2017;19:24.

15 Debette S, et al: Midlife vascular risk factor exposure accelerates structural brain aging and cognitive decline. Neurology 2011;77:461-468.

16 Godin 0, et al: Antihypertensive treatment and change in blood pressure are associated with the progression of white matter lesion volumes: the Three-City (3C)-Dijon Magnetic Resonance Imaging Study. Circulation 2011; 123:266-273.

17 Law MR, Morris JK, Wald NJ: Use of blood pressure lowering drugs in the prevention of cardiovascular disease: meta-analysis of 147 randomised trials in the context of expectations from prospective epidemiological studies. BMJ 2009;338:b1665.

18 Tadic M, Cuspidi C, Hering D: Hypertension and cognitive dysfunction in elderly: blood pressure management for this global burden. BMC Cardiovasc Disord 2016;16:208.

19 Ninomiya T, et al: Midlife and late-life blood pressure and dementia in Japanese elderly: the Hisayama study. Hypertension 2011;58:22-28.

20 Gottesman RF, et al: Midlife hypertension and 20-year cognitive change: the Atherosclerosis Risk in Communities Neurocognitive study. JAMA Neurol 2014;71:1218-1227.

21 Mitchell GF: Arterial stiffness and hypertension: chicken or egg? Hypertension 2014;64:210-214.

-22 Maillard P, et al: Effects of arterial stiffness on brain integrity in young adults from the Framingham Heart Study. Stroke 2016;47:1030-1036.

23 Hajjar I, et al: Roles of arterial stiffness and blood pressure in hypertension-associated cognitive decline in healthy adults. Hypertension 2016;67:171-175.

-24 Pase MP: Modifiable vascular markers for cognitive decline and dementia: the importance of arterial aging and hemodynamic factors. J Alzheimers Dis 2012;32:653-663.

25 Gottesman RF, et al: Associations between midlife vascular risk factors and 25-year incident dementia in the Atherosclerosis Risk in Communities (ARIC) cohort. JAMA Neurol 2017;74:1246-1254.

26 Markus HS: Cerebral perfusion and stroke. J Neurol Neurosurg Psychiatry 2004;75:353-361.

-27 Gorelick PB, et al: Vascular contributions to cognitive impairment and dementia: a statement for healthcare professionals from the American Heart Association/American Stroke Association. Stroke 2011;42:2672-2713.

-28 de Roos A, et al: Magnetic resonance imaging of cardiovascular function and the brain: is dementia a cardiovascular-driven disease? Circulation 2017;135:2178-2195.

29 Henskens LH, et al: Increased aortic pulse wave velocity is associated with silent cerebral small-vessel disease in hypertensive patients. Hypertension 2008;52:1120-1126.

-30 Pase MP, et al: Association of aortic stiffness with cognition and brain aging in young and middle-aged adults: the Framingham Third Generation Cohort Study. Hypertension 2016;67:513-519.

31 Singer J, et al: Arterial stiffness, the brain and cognition: a systematic review. Ageing Res Rev 2014;15:16-27.

-32 Maillard P, et al: Aortic stiffness, increased white matter free water, and altered microstructural integrity: a continuum of injury. Stroke 2017;48:1567-1573.

-33 Burciu RG, et al: Free-water and BOLD imaging changes in Parkinson's disease patients chronically treated with a MAO-B inhibitor. Hum Brain Mapp 2016;37:2894-2903.

-34 Ofori E, et al: Longitudinal changes in free-water within the substantia nigra of Parkinson's disease. Brain 2015; 138(Pt 8):2322-2331.

-35 Wang KL, et al: Wave reflection and arterial stiffness in the prediction of 15-year all-cause and cardiovascular mortalities: a community-based study. Hypertension 2010;55:799-805.

-36 Climie RE, et al: Aortic reservoir characteristics and brain structure in people with type 2 diabetes mellitus; a cross sectional study. Cardiovasc Diabetol 2014;13:143.

-37 Fu C, et al: Comparison of the Mini-Mental State Examination and Montreal Cognitive Assessment executive subtests in detecting post-stroke cognitive impairment. Geriatr Gerontol Int 2017, Epub ahead of print.

-38 Lonie JA, Tierney KM, Ebmeier KP: Screening for mild cognitive impairment: a systematic review. Int J Geriatr Psychiatry 2009;24:902-915.

-39 Nasreddine ZS, et al: The Montreal Cognitive Assessment, MoCA: a brief screening tool for mild cognitive impairment. J Am Geriatr Soc 2005;53:695-699.

-40 Freitas S, et al: Montreal Cognitive Assessment: validation study for mild cognitive impairment and Alzheimer disease. Alzheimer Dis Assoc Disord 2013;27:37-43.

-41 Trzepacz PT, et al: Relationship between the Montreal Cognitive Assessment and Mini-Mental State Examination for assessment of mild cognitive impairment in older adults. BMC Geriatr 2015;15:107.

-42 Suleman R, et al: Association between central blood pressure, arterial stiffness, and mild cognitive impairment. Clin Hypertens 2017;23:2. 